\title{
Discovery of Dark Space
}

\author{
Alexander Alecsandrovich Antonov \\ Independent Researcher, Kiev, Ukraine \\ Email: telan@bk.ru
}

How to cite this paper: Antonov, A.A. (2018) Discovery of Dark Space. Journal of Modern Physics, 9, 14-34.

https://doi.org/10.4236/jmp.2018.91002

Received: October 6, 2017

Accepted: January 6, 2018

Published: January 9, 2018

Copyright $\odot 2018$ by author and Scientific Research Publishing Inc. This work is licensed under the Creative Commons Attribution International License (CC BY 4.0).

http://creativecommons.org/licenses/by/4.0/

\section{Open Access}

\begin{abstract}
It is currently a generally accepted opinion in astrophysics that the phenomenon of dark matter and dark energy can be explained only by existence of microcosm contents unknown to us. This assumption seems to be quite reasonable, since the Monoverse hypothesis following from the postulate about non-exceedance of the speed of light in the special theory of relativity, leaves no place for dark matter and dark energy in macrocosm. However, the assumption is erroneous, as dark matter and dark energy are actually invisible parallel universes in six-dimensional space, as shown in the article. Therefore, they can be detected only during research of macrocosm. Research of the phenomenon of dark matter and dark energy is presented in the article. Moreover, it is performed using previously unknown experiments that have allowed proof of general scientific principle of physical reality of concrete imaginary numbers. In the special theory of relativity, this principle has refuted the postulate of light speed non-exceedance. It has also allowed adjustment of relativistic formulas. It follows from the adjusted relativistic formulas that we live in a hidden Multiverse. The article explains the nature of mutual invisibility of parallel universes of the hidden Multiverse and proves verifiability of the hypothesis of the hidden Multiverse. The hypothesis has explained the nature of dark matter and dark energy as well as given an idea where antimatter is and why it does not annihilate with matter. It has also explained where tachyons are and why they do not violate the principle of causality. According to the hypothesis, the hidden Multiverse is connected to other Multiverses through portals, forming dark space. Multiverses of dark space together with the hidden Multiverse form a Hyperverse.
\end{abstract}

\section{Keywords}

Imaginary Numbers, Dark Matter, Dark Energy, Dark Space, Multiverse, Hyperverse

\section{Introduction}

In the twentieth century, two extremely important scientific discoveries were 
made in astrophysics [1] [2] [3] [4]. The first of them was made in 1932-1933 by Jan Hendrik Oort [5] and Fritz Zwikky [6] and called dark matter. The second one, called dark energy, was made in 1998-1999 by Saul Perlmutter [7], Brian P. Schmidt [8] and Adam G. Riess [9] who were awarded the Nobel Prize. The total mass-energy of dark matter and dark energy more than twenty times exceeded the total mass-energy of our visible universe.

However, the discoveries turned out to be quite incomprehensible. In any range of electromagnetic waves, dark matter and dark energy are absolutely invisible. Besides, none of chemical elements known to us has been detected in dark matter and dark energy. Consequently, these discoveries have presumably discredited such fundamental scientific terms as "atom", "molecule" and "matter" and threw back the development of science by millennia.

Therefore, over the past decades, much attention has been paid to finding an explanation of the phenomenon of dark matter and dark energy, and intensive scientific research has been done. However, the phenomenon has not been explained.

This situation has naturally made us wonder what the reason was for such a result. What's the mistake? What might be the direction of possible alternative research according to Albert Einstein: "Insanity. doing the same thing over and over again and expecting different results."?

The reason for inexplicability of the phenomenon is that names of these scientific discoveries have been understood too literally. Yet, it was impossible to give them more specific names because of the extreme incomprehensibility of the subject of discovery. Nevertheless, the names of the discoveries and facts known about them have suggested that explanation of dark matter and dark energy should be sought somewhere in microcosm, in some sort of its new structure. Moreover, it seemed that there was nowhere else to look for explanation of dark matter and dark energy as according to the hypothesis of Monoverse following from the principle of light speed non-exceedance ${ }^{1}$ in the special theory of relativity (STR).

However, these assumptions have been erroneous, since dark matter and dark energy are actually entities of macrocosm, as shown below. These are invisible parallel universes of a Multiverse ${ }^{2}$. They are invisible because the structure of the Multiverse is described by physically real hyper-complex numbers.

Physical reality of complex, and even more so, hyper-complex numbers has so far been completely unfeasible, since STR denies physical reality of imaginary numbers $^{3}$ due to inability to explain it by the principle of light speed non-exceedance.

\footnotetext{
${ }^{1}$ Which, as is often asserted (but never proved), is supposedly identical to the principle of light speed constancy, i.e. being another formulation of the second postulate of the STR. But in any science other than STR, the use of several different formulations for the same axioms, postulates, theorems or regularities is inadmissible.

${ }^{2}$ Since, despite their infinity, they never intersect.

${ }^{3}$ Physical reality of imaginary numbers, as well as real numbers, is actually reasonable to speak of only in respect of concrete numbers provided with references to units of measurement used for the corresponding parameters of physical entities or processes.
} 
It would seem to have a reason, since physical reality of imaginary numbers discovered five hundred years ago by Scipione del Ferro, Niccolò Fontana Tartaglia, Gerolamo Cardano, Lodovico Ferrari and Rafael Bombelli [10], has remained unproved despite considerable efforts. Besides, this problem turned out [11] to have no purely mathematical solution, as it has actually been interdisciplinary. Therefore, according to the statement by Oliver Heaviside "Mathematics is an experimental science" the solution shall be supported by experiments.

The article describes the experiments, proving physical reality of imaginary numbers and allowing, in the long run, explanation of the phenomenon of dark matter and dark energy.

\section{Proof of Physical Reality of Imaginary Numbers}

Let's begin the research of the phenomenon of dark matter and dark energy with the proof of physical reality of imaginary numbers, as further presentation is based on the general scientific principle. Only one of its proofs is given [12] [13] [14] [15] [16], since it is convincing enough. This proof, as well as other well-known proofs [11] [17]-[26], unlike the axiomatic approach popular in modern physics, has been obtained as a result of conducting previously unknown experiments being the only decisive argument ${ }^{4}$ in science, rather than using certain postulates (i.e., unproved assumptions). Herewith, only real experiments are meant and never the so-called "mental experiments" in the STR, which are nothing but reasoning.

The proof involves the Ohm's law ${ }^{5}$ which is known to all educated people. However, Ohm's law is applied only in later interpretation proposed by Charles Proteus Steinmetz [27], which is known as a symbolic method for calculating linear electric circuits. The proof based on such an interpretation of the Ohm's law is the simplest and most comprehensible. It is as follows. Resistance of resistor $R$ is assumed to be equal to a real number $R$, the value of which does not depend on frequency $\omega$ of sinusoidal voltage applied thereto. Reactance of inductor $L$ is assumed to be equal to a positive imaginary number ${ }^{6} j \omega L$, the value of which does depend on frequency $\omega$ of voltage applied thereto. Reactance of

\footnotetext{
${ }^{4}$ Driven by the same reasons, Cardinal Richelieu ordered in the Thirty Years' War to inscribe upon cannons the following text: "Ultima ratio regum" i.e. the last argument of king. The last argument of a scientist is experiments.

${ }^{5}$ In 1826 Georg Simon Ohm discovered the law that now bears his name as a result of nine years of experimental research. Its complexity was reflected by the fact that there were no electric measuring instruments at that time. Moreover, scientific research in physics was done differently that it's done today. Famous physicist Alexander Grigorievich Stoletov wrote in this regard: “...physics especially tempted natural philosophers. What a favorable theme were electrical phenomena for the most riotous imaginations... Attractive and vague deductions were in the foreground: hard work of experimenter and exact mathematical analysis were not honored; they seemed superfluous and harmful in the study of nature...”. Therefore, contemporaries of Ohm gave to his discovery quiet a lukewarm support. In $1828 \mathrm{Ohm}$ was even fired by personal order of Minister of Education for publishing his physics discoveries. A senior official believed that the use of mathematics in physics was unacceptable.

${ }^{6}$ In electrical engineering, unlike mathematics, the imaginary unit $\sqrt{-1}$ is usually denoted by the letter $j$, since $i$ denotes electric current.
} 
capacitor $C$ is assumed to be equal to a negative imaginary number $-j / \omega C$, the value of which also depends on frequency $\omega$ of voltage applied thereto.

Therefore, resistance $Z(j \omega)$ of any electric $L C R$-circuit will be equal to a concrete complex number, the value of which depends on frequency $\omega$ of applied voltage. If imaginary inductive and capacitive reactances are physically existent, the magnitude of current flowing through the electric $L C R$-circuit would depend on frequency $\omega$ of applied voltage and vice versa. Otherwise, they would be imaginary, i.e. physically nonexistent both by name and nature.

Consequently, an experiment determining whether imaginary inductive and capacitive reactances and hence imaginary numbers in general are physically existent is very simple. The only thing to do is to change frequency of sinusoidal voltage applied to any electric $L C R$-circuit and check whether magnitude of current flowing through it changes.

Millions of electric and radio engineers all over the world conduct such experiments in the course of their daily work. Therefore, they all know that magnitude of current flowing through electric $L C R$-circuit changes with frequency of voltage applied thereto. These changes can be seen on oscilloscope screen. Moreover, instruments for measuring frequency characteristics registering such changes have been industrially produced many decades.

Using this knowledge, engineers create, by changing frequency characteristics of electric $L C R$-circuits, a variety of filters, without which existence of radio engineering, telecommunication, television, radiolocation, radio navigation and other exact sciences would not have been possible.

Moreover, if inductive and capacitive reactances were physically nonexistent, resonance discovered by Galileo di Vincento Bonaiuti de'Galilei ${ }^{7}$ [28] in 1602, without which radio engineering, telecommunication, television, radiolocation, radio navigation also could not exist, would also have been impossible in electric $L C R$-circuits.

Consequently, any experiment involving the Ohm's law in the interpretation of Steinmetz definitely proves physical reality of imaginary numbers, and thus refutes and obviates the principle of light speed non-exceedance.

It is also notable that as the theory of Charles Proteus Steinmetz [27] was developed in 1893 and the STR [29] [30] [31] was developed by Joseph Larmor [32], Nobel Prize winner Hendrik Antoon Lorentz [33], Jules Henri Poincaré [34] and Nobel Prize winner Albert Einstein [35] in 1897-1905, the existing interpretation of the STR could be refuted by the above arguments even before its creation.

And if authors of the STR could be excused for not knowing this, nowadays everyone has to know this.

\section{Adjustment of the Special Theory of Relativity}

However, everyone doesn't know this. Therefore, now as a hundred years ago the existing version of the STR has denied physical reality of imaginary numbers,

${ }^{7}$ However, definitely not in electric circuits. 
referring to the principle of light speed non-exceedance postulated in the STR. The principle is substantiated by the following arguments. According to the Lorentz-Einstein formula

$$
m=\frac{m_{0}}{\sqrt{1-(v / c)^{2}}}
$$

where $m_{0}$ is the rest mass of a moving entity (e.g. elementary particle);

$m$ is the relativistic mass of a moving entity;

$v$ is the velocity of a physical entity;

$c$ is the speed of light.

$\lim _{v \rightarrow c-0} m(v)=+\infty$. This means that relativistic mass $m$ assumes real values approaching to infinitely large values at velocities of physical entities $v$ approximate to the speeds of light $c$, given that $v<c$. Hence, it follows that overcoming of light speed barrier requires infinite energy. Authors of the STR concluded that the overcoming was impossible. Therefore, they believed that there was nothing behind the barrier and people lived in a Monoverse ${ }^{8}$. In this regard, imaginary mass has allegedly no physical sense at $v>c$ in the Formula (1).

However, such quite convincing, at first sight, arguments are refuted even by the simplest everyday events. For example, inability to overcome a barrier in the form of a wall dividing adjacent rooms of your home does not mean that there is no way to get into the next room through a door. It neither means that the adjacent room does not exist or contains nothing. Undoubtedly, the universe is organized more intricate than our home. It actually contains many physical entities and processes still unknown to us, as well as their peculiar capabilities. Consequently, reasoning about Monoverse existence by authors of the STR seems unconvincing.

Therefore, arguments for the principle of light speed non-exceedance have not been considered convincing by all physicists. In 2011 OPERA Collaboration published results of the sensational experiment [36], which supposedly refuted the principle of light speed non-exceedance and thereby proved physical reality of imaginary numbers. However, in half a year the OPERA experiment was refuted by the ICARUS experiment [37].

Even earlier, in 2008-2010 there were alternative experiments ${ }^{9}$ [17]-[22] which successfully solved the problem of proving physical reality of imaginary numbers by studying oscillatory processes in linear electric $L C R$-circuits. And since the experiments can be repeated and verified in any electrical engineering and electronic laboratory, they are quite reliable and verifiable. The same can't be said for unsuccessful OPERA experiment. It turned to be needless, as it attempted to solve the already-solved issue.

Thus, imaginary relativistic mass $m$ is physically real at supersonic speeds,

${ }^{8}$ That is the only universe existing in nature.

${ }^{9}$ Much earlier, Steinmetz proposed his own interpretation of the Ohm's law, which, as shown above, also allowed verification of physical reality of imaginary numbers. 
given that $v>c$ and, therefore, has to be explained [38] [39]. However, the Formula (1) does not allow this, since it is valid only in the range $0 \leq v<c$, whereas in the range $c \leq v<\infty$ it does not correspond to the first postulate of the STR. Therefore, to change the range of velocities $0 \leq v<c$ the Formula (1) can be adjusted as follows

$$
m=\frac{m_{0} i^{q}}{\sqrt{1-(v / c-q)^{2}}}=\frac{m_{0} i^{q}}{\sqrt{1-(w / c)^{2}}}
$$

where $q=\lfloor v / c\rfloor$ is the discreet "floor" function of argument $v / c$;

$v$ is the velocity measured from our tardyon universe, which shall therefore be called tardyon velocity;

$w=v-q c$ is the local velocity for each universe, which can take values only in the range $0 \leq w<c$.

Other relativistic formulas of the STR can be adjusted in a similar manner.

Albert Einstein did not exclude the adjustment of the STR in future. He wrote: "No single idea, which I would be sure that it will stand the test of time". He also asserted: "We can't solve problems by using the same kind of thinking we used when we created them".

\section{Hypothesis of the Hidden Multiverse}

Physical sense of the Formula (2) can be explained as follows [40] [41] [42] [43]. Different integral ${ }^{10}$ values of the quantity $q$ in the Formula (2) correspond to different parallel universes: the value $q=0$ corresponds to our universe (as $i^{0}=1$ ), and the value $q=1$ corresponds to another universe (as $i^{1}=i$ ), in which there are tachyons and which shall be, for definiteness, referred to as a tachyon. For similar reasons, our universe is called a tardyon. Tachyon universe is behind the horizon of events and therefore, is invisible for us. For tachyon universe $c \leq v<2 c$. Consequently, the Formula (2), in contrast to Formula (1), corresponds to an invisible Multiverse, which shall be referred to as a hidden Multiverse.

However, the hidden Multiverse could contain more than two parallel universes. It is shown below that they could actually be even more than twenty. In this regard, the value $q=2$ in the hidden Multiverse would correspond to tardyon antiverse (for which $i^{2}=-1$ ), the value $q=3$ would correspond to tachyon antiverse (for which $i^{3}=-i$ ), the value $q=4$ would correspond to another tardyon universe (for which $i^{4}=1$ ), the value $q=5$ would correspond to another tachyon universe (for which $i^{5}=i$ ), etc.

But where are these parallel universes, if they do not intersect? In what space?

Space is determined by an extra dimension in the Formula (2). It implies that space of such a hidden Multiverse is four-dimensional. Three dimensions $x, y, z$ of space determine mutual spatial position of material contents of each

${ }^{10} \mathrm{~A}$ quantity $q$ takes non-integer value in the portals considered below, in which it varies (from entry to exit) from one integer value to another integer value corresponding to parallel universes between which there are portals. 
parallel universe, and the fourth dimension $q$ in the hidden Multiverse determines mutual spatial position of the parallel universes. Therefore, such a four-dimensional structure can be described by a complex number ${ }^{11}$ $f_{q}(x, y, z)+i q$.

Besides, due to continuous drifting all parallel universes in the fourth dimension of the hidden Multiverse can touch their adjacent universes and even slightly penetrate into them generating many portals ${ }^{12}$ [44] [45]. They are denoted by single two-sided arrows in Figures 1-3. Portals on Earth are so-called anomalous zones [46] that can be everywhere on, above and below its surface. Adjacent universes can exchange their material contents through the portals. Therefore, mass of parallel universes of the hidden Multiverse should have been substantially averaged over billions of years of their existence.

Taking into account this circumstance, we will analyze structures of the hidden Multiverse, which can have the form shown in Figures 1-3, are. As can be seen, the structure shown in Figure 1 has the form of a close screw collar, and in Figure 2 and Figure 3 it presented as an open screw collar.

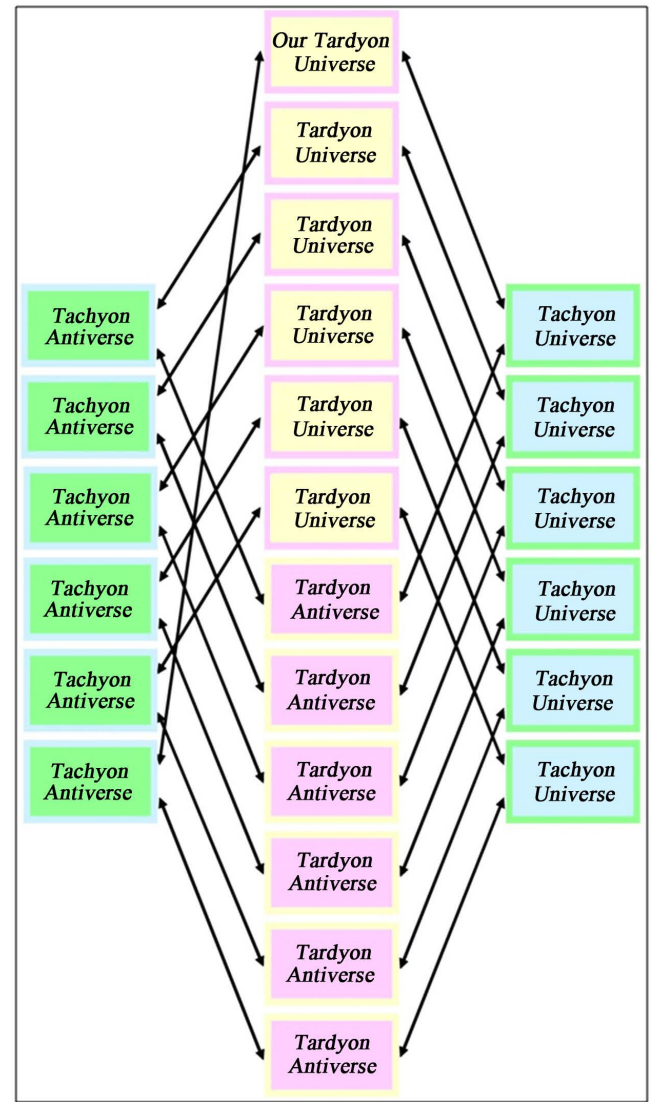

Figure 1. (Color online) Collar structure of the hidden Multiverse corresponding to the principle of physical reality of imaginary numbers.

${ }^{11}$ Let us recall that concrete imaginary numbers correspond to physical entities which could not be seen, heard or otherwise felt.

${ }^{12}$ Which have nothing to do with "wormholes" in the general theory of relativity. 


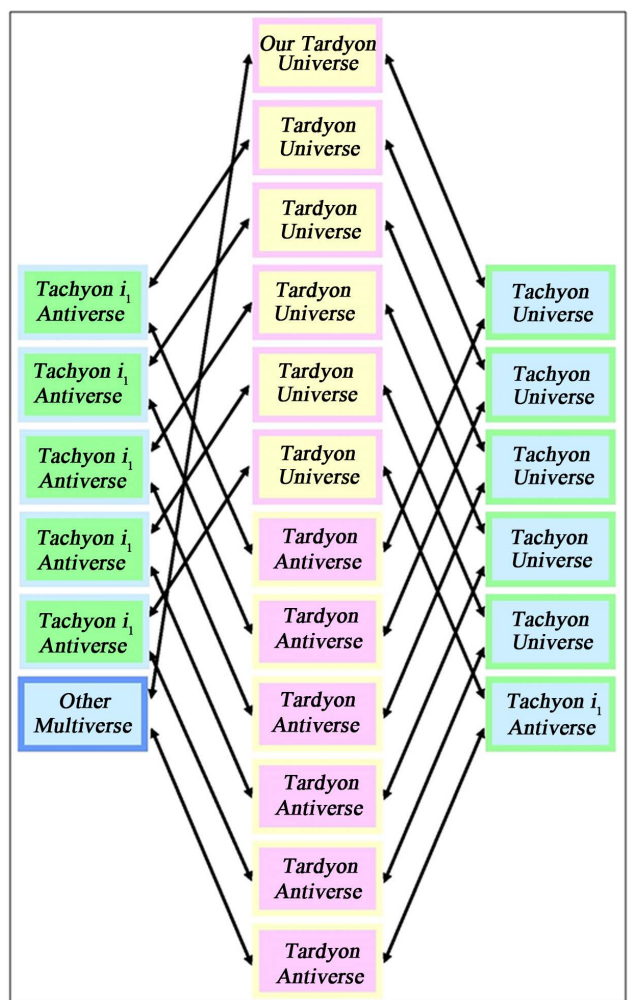

Figure 2. (Color online) Another structure of the hidden Multiverse corresponding to the principle of physical reality of imaginary numbers.

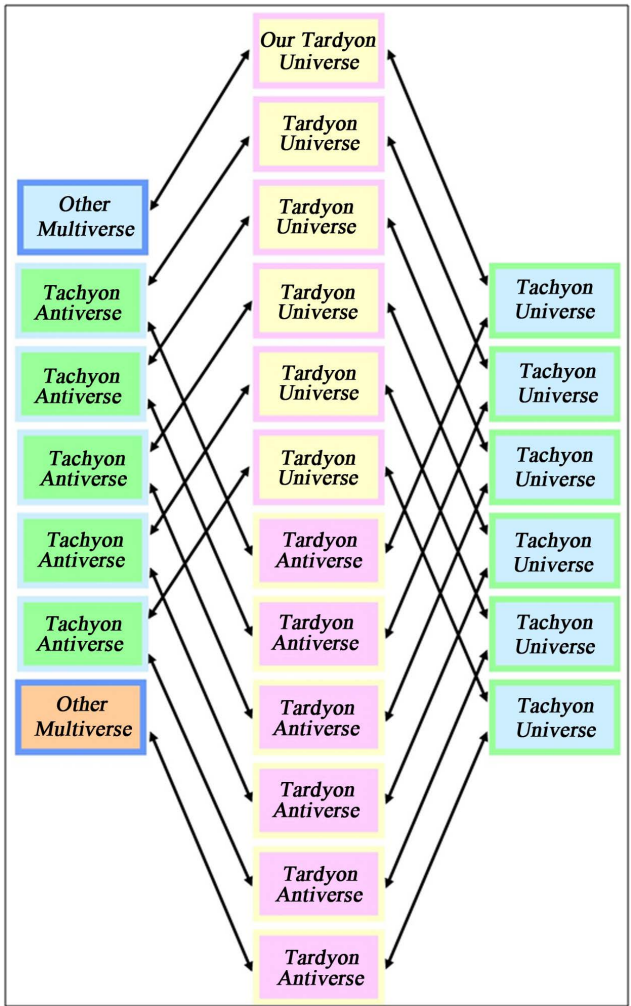

Figure 3. (Color online) One more structure of the hidden Multiverse corresponding to the principle of physical reality of imaginary numbers. 
The structure shown in Figure 1 might well be the only one in nature, since it fully closes on itself. And even if there are any other universes or Multiverses, inhabitants of such a hidden Multiverse will never know about it, since they can't leave their hidden Multiverse.

The structures of the hidden Multiverse depicted in Figure 2 and Figure 3 do not close on themselves. Such hidden Multiverses are not unique, since they can't have edges, behind which there is something unknown. Therefore, they are invariably connected through portals to other Multiverses: two different Multiverses (as in Figure 2) or twice to the same Multiverse (as in Figure 3). Thus, they form a larger structure, which shall be called a Hyperverse.

\section{Explanation of Dark Matter and Dark Energy}

Now the phenomenon of dark matter and dark energy is explicable. It has been actually inexplicable only within the Monoverse hypothesis. There have been so far persistent, but unsuccessful attempts to explain the phenomenon using the hypothesis.

Therefore, seeking explanation of the phenomenon of dark matter and dark energy within other hypotheses of structure of the world we live in is not only justified but even necessary. The explanation becomes not only possible, but also obvious within the hypothesis of the hidden Multiverse. It is actually quite clear that:

- dark matter and dark energy correspond to other, besides ours, parallel universes of the hidden Multiverse;

- dark matter corresponds to parallel universes of the hidden Multiverse adjacent to ours;

- dark energy corresponds to the rest universes of the hidden Multiverse, besides our and adjacent universes.

Thus, dark matter and dark energy are other names for the rest parallel universes of the hidden Multiverse, besides ours.

\section{Analysis of WMAP and Planck Spacecraft Data}

Data obtained by the WMAP [47] and Planck [48] spacecrafts allow substantial adjustment of structure of the hidden Multiverse. Thus, according to the measurements of the WMAP spacecraft, the entire universe (in fact, the entire hidden Multiverse) is composed of $4.6 \%$ baryonic matter, $22.4 \%$ of dark matter and $73.0 \%$ of dark energy. According to the later measurements of the Planck spacecraft, the entire universe (again, in fact, the entire hidden Multiverse) is composed of $4.9 \%$ baryonic matter, $26.8 \%$ of dark matter and $68.3 \%$ of dark energy.

These data, therefore, suggest that [49]-[59]:

- According to WMAP data the hidden Multiverse contains 100\%/4.6\% $=21.7$ universes, whereas according to Planck data it consists of $100 \% / 4.9 \%=20.4$, i.e. $20 \cdots 22$ parallel universes;

- According to WMAP data dark matter contains $22.4 \% / 4.6 \%=4.9$ uni- 
verses, whereas according to Planck data it consists of $26.8 \% / 4.9 \%=5.5$, i.e. $5 \cdots 6$ parallel universes;

- According to WMAP data dark energy contains $73.0 \% / 4.6 \%=15.9$ universes, whereas according to Planck data it consists of $68.3 \% / 4.9 \%=13.9$, i.e. $14 \cdots 16$ parallel universes.

The structure of the hidden Multiverse allows us to explain not only the phenomenon of dark matter and dark energy, but also where tachyons are [60]-[65]. They turn out to be in numerous tachyon universes and antiverses. Therefore, the assertion that they cannot exist, since they allegedly violate the principle of causality, is erroneous. In fact, this is not so, because tachyons and tardyons are in different parallel universes.

The structure of the hidden Multiverse also convincingly explains where antimatter is [64] [66]-[72]. It can be found in antiverses. There are a lot of pairs of universes-antiverses. Universes and antiverses do not annihilate, because tardyon and tachyon universes and antiverses are interspersed in the structure of the hidden Multiverse.

\section{Adjustment of the Special Theory of Relativity (Continuation)}

However, the above explanation of the structure of the hidden Multiverse, which is apparently quite consistent, is not entirely perfect. The thing to be noticed is that our tardyon universe should have five or six adjacent parallel universes, rather than two (one tachyon universe and one tachyon antiverse), as in Figures 1-3. This difference is too great to be referred to the error of WMAP and Planck measurements.

This means that another explanation is needed. It is as follows. There is an error in arguments referring to Figures 1-3. The error is due to the assumption that there is only one extra dimension in the hidden Multiverse. Therefore, it supposedly corresponds to physically real complex numbers $f_{q}(x, y, z)+i q$ containing only one imaginary unit.

There should be three extra dimensions $q, r, s$ for explanation of the structure of the hidden Multiverse in which our tardyon universe can have six other adjacent universes: three tachyon universes and three tachyon antiverses. Consequently, the structure of the hidden Multiverse should correspond to physically real quaternions $\sigma+i_{1} \omega_{1}+i_{2} \omega_{2}+i_{3} \omega_{3}$ [73], just containing three imaginary units $i_{1}, i_{2}, i_{3}$, which are related as follows

$$
\begin{gathered}
i_{1}^{2}=i_{2}^{2}=i_{3}^{2}=1 \\
i_{1} i_{2} i_{3}=i_{2} i_{3} i_{1}=i_{3} i_{1} i_{2}=-1 \\
i_{1} i_{3} i_{2}=i_{2} i_{1} i_{3}=i_{3} i_{2} i_{1}=1
\end{gathered}
$$

Therefore, the adjusted relativistic Formula (2) given above should be adjusted once again as follows 


$$
m=\frac{m_{0}\left(i_{1}\right)^{q}\left(i_{2}\right)^{r}\left(i_{3}\right)^{s}}{\sqrt{1-[v / c-(q+r+s)]^{2}}}=\frac{m_{0}\left(i_{1}\right)^{q}\left(i_{2}\right)^{r}\left(i_{3}\right)^{s}}{\sqrt{1-(w / c)^{2}}}
$$

where $q$ is the total number of parallel universes, penetration into which is made through portals, corresponding to the imaginary unit $i_{1}$, with increasing distance from our tardyon universe;

$r$ is the total number of parallel universes, penetration into which is made through portals, corresponding to the imaginary unit $i_{2}$, with increasing distance from our tardyon universe;

$s$ is the total number of parallel universes, penetration into which is made through portals, corresponding to the imaginary unit $i_{3}$, with increasing distance from our tardyon universe;

$q+r+s$ is the parameter characterizing the degree of remoteness of a universe with $q, r, s$ coordinates from our tardyon universe;

$v$ is the velocity measured from our tardyon universe, which, therefore, can be called tardyon velocity;

$c$ is the speed of light;

$w=v-(q+r+s) c$ is the local velocity for corresponding universe, which can take values only in the range $0 \leq w<c$.

Other relativistic formulas can be adjusted similarly, for example

$$
\begin{aligned}
\Delta t & =\Delta t_{0}\left(i_{1}\right)^{q}\left(i_{2}\right)^{r}\left(i_{3}\right)^{s} \sqrt{1-[v / c-(q+r+s)]^{2}} \\
& =\Delta t_{0}\left(i_{1}\right)^{q}\left(i_{2}\right)^{r}\left(i_{3}\right)^{s} \sqrt{1-(w / c)^{2}} \\
l & =l_{0}\left(i_{1}\right)^{q}\left(i_{2}\right)^{r}\left(i_{3}\right)^{s} \sqrt{1-[v / c-(q+r+s)]^{2}} \\
& =l_{0}\left(i_{1}\right)^{q}\left(i_{2}\right)^{r}\left(i_{3}\right)^{s} \sqrt{1-(w / c)^{2}}
\end{aligned}
$$

where $\Delta t_{0}$ is the rest time of a moving entity;

$\Delta t$ is the relativistic time of a moving entity;

$l_{0}$ is the rest length of a moving entity;

$l$ is the relativistic length of a moving entity;

It can also be argued that physical reality of quaternions proves physical reality of other hyper-complex numbers.

\section{Discovery of Dark Space}

Figure 4 presents a quaternion structure of the hidden Multiverse [74] in the form of a close screw collar corresponding to Formulas (6), (7) and (8). This structure is, for simplicity, shown as an open screw collar. Therefore, our tardyon universe is figuratively depicted twice in the structure: as the beginning and the end of reference of parallel universes.

As can be seen, in this structure, in contrast to the structure shown in Figure 1 , six parallel universes (three tachyon universes $i_{1}, i_{2}, i_{3}$ and three tachyon antiverses $i_{1}, i_{2}, i_{3}$ ) are adjacent to our universe (and other tardyon universes). Therefore, such a six-dimensional structure can be described by a quaternion 


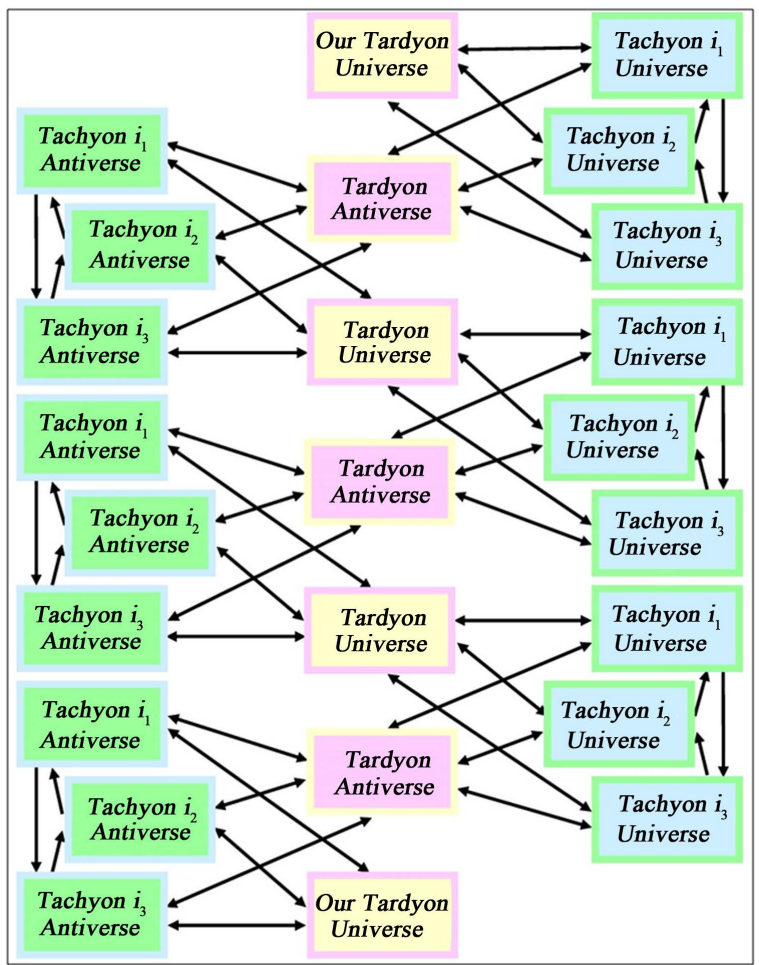

Figure 4. (Color online) Collar structure of the hidden Multiverse corresponding to the principle of physical reality of quaternions.

$f_{q, r, s}(x, y, z)+i_{1} q+i_{2} r+i_{3} s$, where the function $f_{q, r, s}(x, y, z)$ corresponds to the distribution of material contents in the corresponding parallel universe, and the expression $i_{1} q+i_{2} r+i_{3} s$ determines coordinates of this universe.

Another difference of this structure from the one shown in Figure 1 is that it contains unidirectional portals corresponding to Formulas (4) and (5), in addition to bidirectional portals corresponding to Formula (3). Principle of unidirectional portals functioning is explained in detail in [75].

However, as can be seen, close collar structure shown in Figure 4 contains twenty-four parallel universes, rather than twenty to twenty-two parallel universes, as follows from the data of the WMAP and Planck spacecrafts.

Therefore, quaternion structure of the hidden Multiverse corresponding to the WMAP and Planck data, as well as the structures shown in Figures 1-3, has edges in points of missing universes, which are connected to other Multiverses through the portals. And these other Multiverses are a new astrophysical entity, referred to as dark space. Together with the hidden Multiverse they form a Hyperverse. Possible structures of the hidden Multiverse that correspond to the WMAP and Planck data, taking into account existence of dark space, are shown in Figure 5-9.

Figure 5 presents a quaternion structure of the hidden Multiverse containing twenty parallel universes. This hidden Multiverse is connected to two other different Multiverses (to one of them three times), with which it forms a Hyperverse. These other Multiverses can presumably have structures different from the 


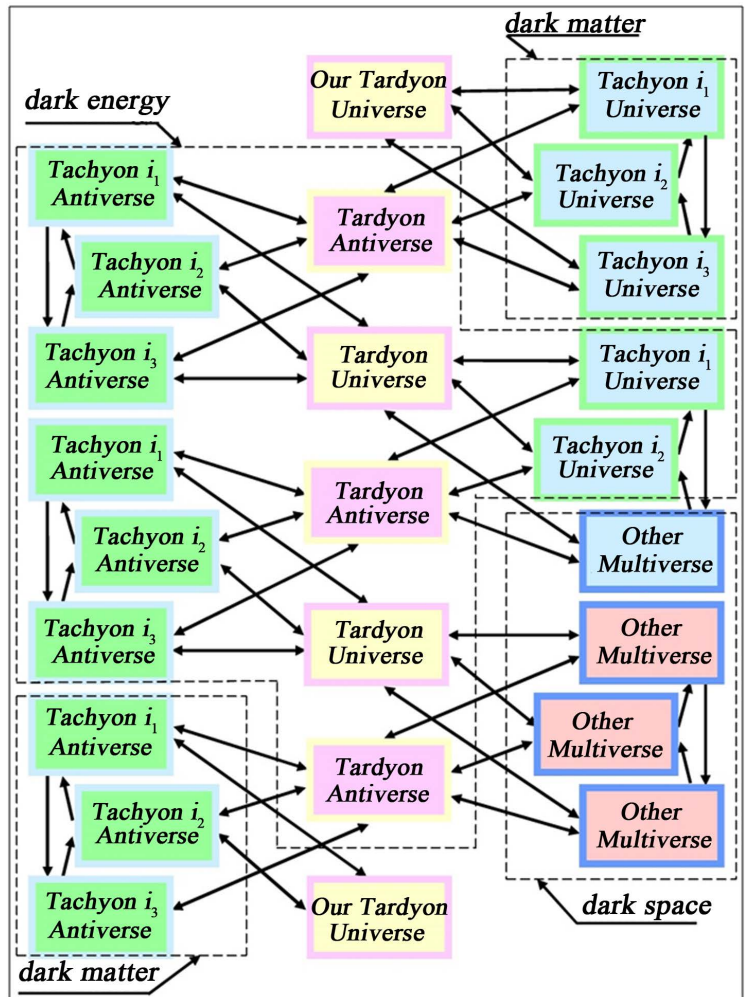

Figure 5. (Color online) Twenty universe structure of the hidden Multiverse corresponding to the principle of physical reality of quaternions.

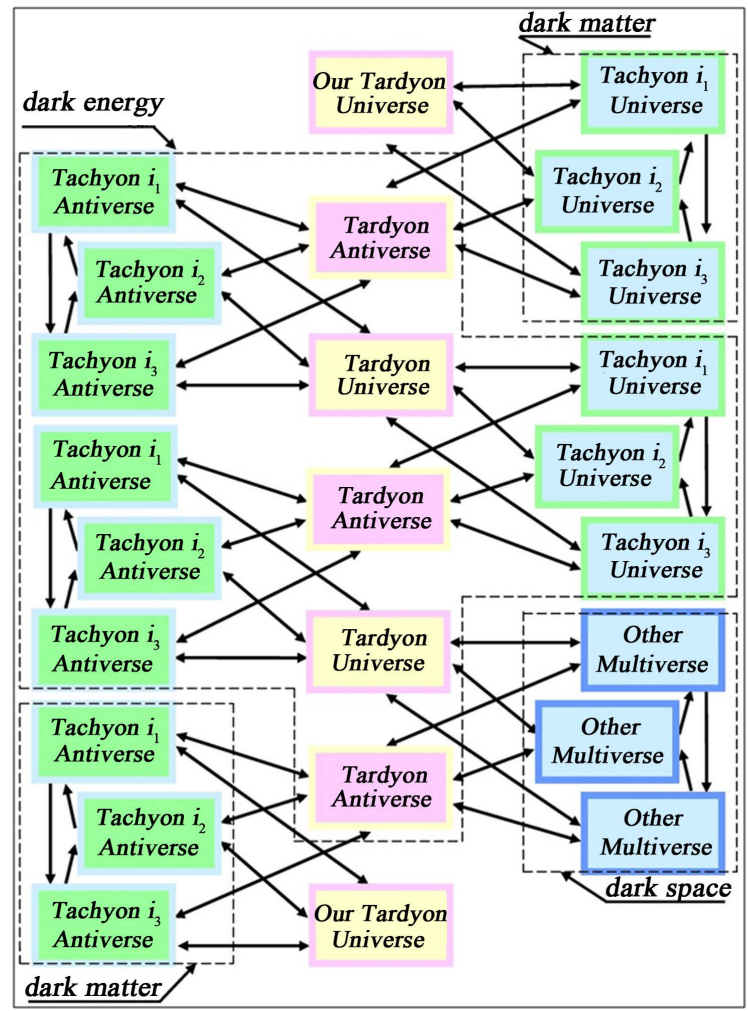

Figure 6. (Color online) Twenty-one universe structure of the hidden Multiverse corresponding to the principle of physical reality of quaternions. 


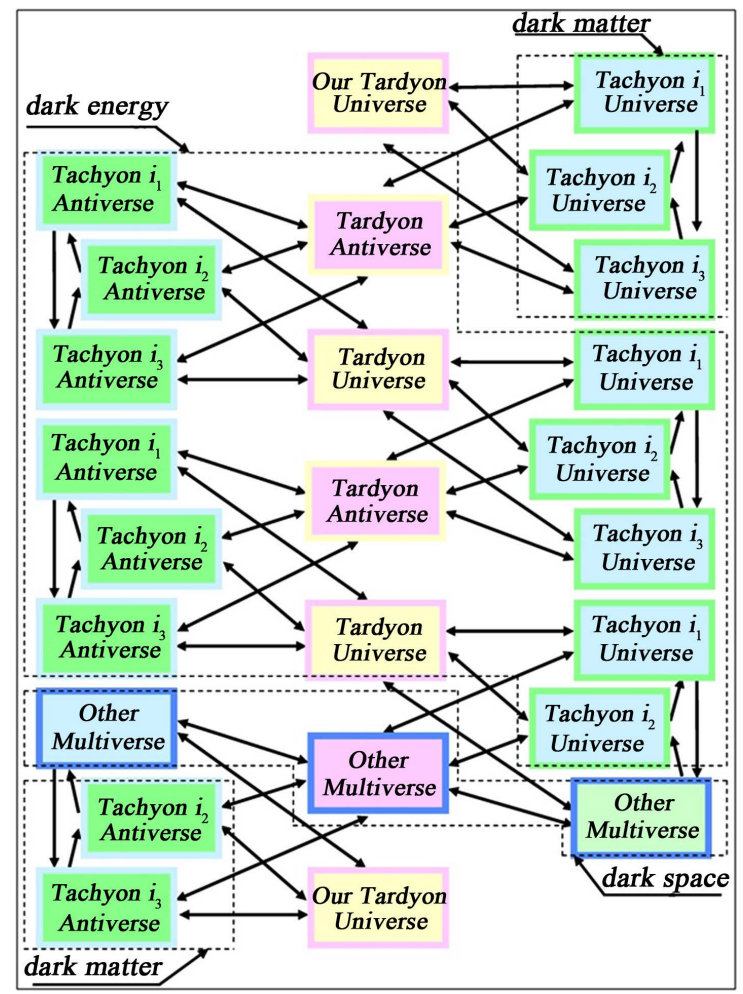

Figure 7. (Color online) Another twenty-one universe structure of the hidden Multiverse corresponding to the principle of physical reality of quaternions.

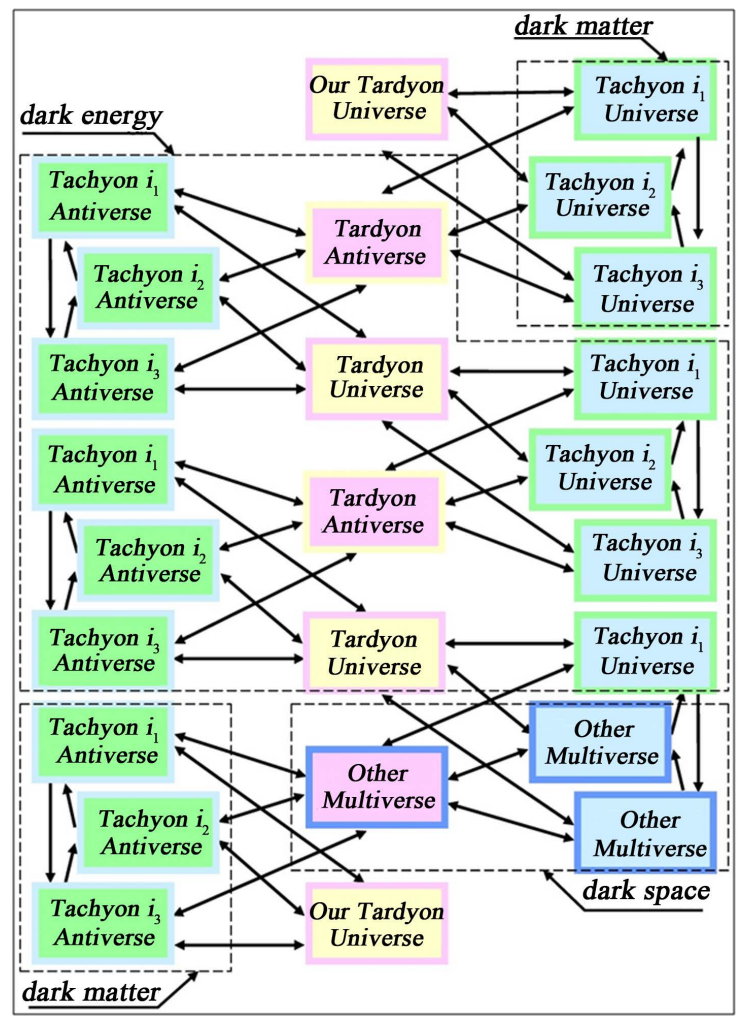

Figure 8. (Color online) One more twenty-one universe structure of the hidden Multiverse corresponding to the principle of physical reality of quaternions. 


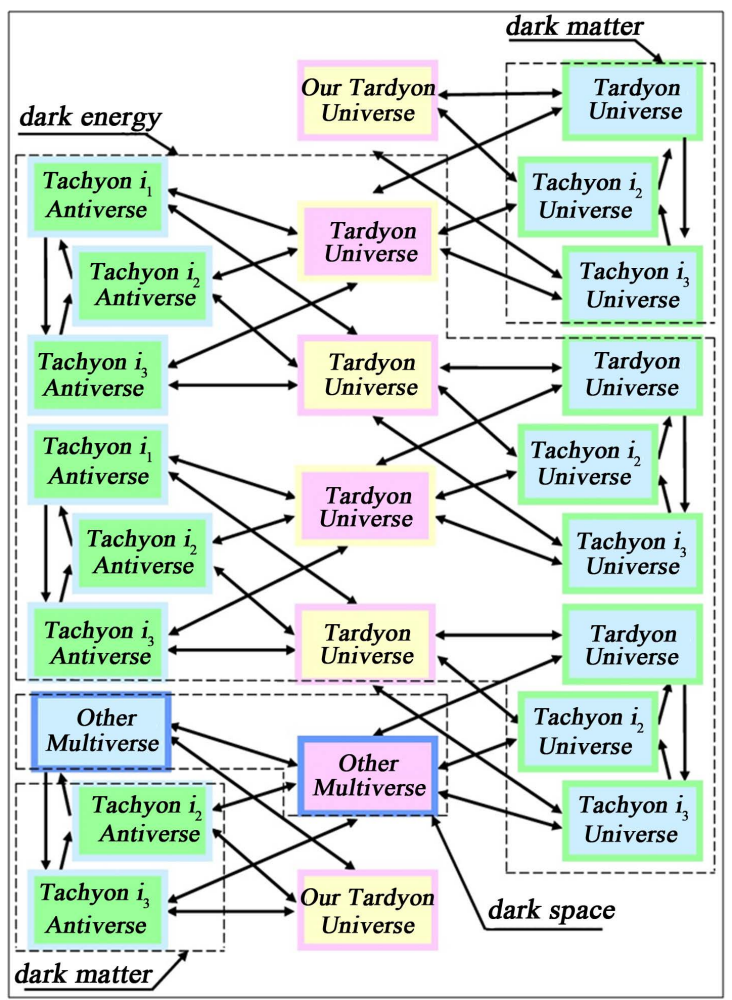

Figure 9. (Color online) Twenty-two universe structure of the hidden Multiverse corresponding to the principle of physical reality of quaternions.

structure of the hidden Multiverse. For example, they can have an octave structure or the structure shown in Figures 1-3 that corresponds to the principle of physical reality of complex numbers.

It is shown in Figure 5 and below which parallel universes form dark matter and which parallel universes form dark energy. Other Multiverses that are not contained in the hidden Multiverse concerned, but contained in a Hyperverse and together form dark space, are also shown. Dark space is in all respects even darker than dark matter and dark energy, i.e. invisible and incomprehensible (or rather, absolutely unstudied). Physical manifestation of dark space, unlike of dark matter and dark energy, has not yet been recorded, because universes referring thereto are even farther away from our tardyon universe than universes of dark energy. Dark space has been discovered only due to mathematical processing of data from WMAP and Planck spacecrafts, using an adequate interpretation of the data.

Now let's distract ourselves a little. Since in all figures our universe is called tardyon, a question could arise a long time ago-why it is not called otherwise. Why is it not called tachyon universe or an antiverse or tardyon antiverse? And now this question can already be answered. As can be seen from Figures 5-9, only tardyon universes and antiverses can have five or six neighboring universes, which is consistent with WMAP and Planck data. As for the tardyon antiverses, they do not differ in their astrophysical functional properties from the tardyon universes. 
Figure 6 shows a quaternion structure of the hidden Multiverse containing twenty-one parallel universes. This hidden Multiverse is connected (three times) to another Multiverse, together with which it forms a Hyperverse.

Figure 7 presents another quaternion structure of the hidden Multiverse containing twenty-one parallel universes. This hidden Multiverse is connected to three different Multiverses, together with which it forms a Hyperverse.

Figure 8 shows one more quaternion structure of the hidden Multiverse containing twenty-one parallel universes. This hidden Multiverse is connected to two other different Multiverses (to one of them twice), together with which it forms a Hyperverse.

Finally, Figure 9 presents a quaternion structure of the hidden Multiverse containing twenty-two parallel universes. This hidden Multiverse is connected to two other different Multiverses, together with which it forms a Hyperverse.

The structures of the hidden Multiverse shown in Figure 5, Figure 6 and Figure 8, where our tardyon universe has six neighboring parallel universes, can be further explained by the Figure 10(a). This figure shows relative spatial location of our universe and universes of dark matter, dark energy, dark space. Apparently, right next to our universe there are universes of dark matter. Behind them there are universes of dark energy. And farthest off from us the Multiverses of dark space are located. And all of them are invisible to us, since they exist in other dimensions. In other words, next to us and around us there another world is not only visible, but also invisible and unknown to us.

And to the structures of the hidden Multiverse shown in Figure 7 and Figure 9, in which our tardyon universe has five neighboring parallel universes, corresponds to Figure 10(b). In this figures, as can be seen, in addition to those shown in Figure 10(a) connections of our universe's to the dark matter, dark

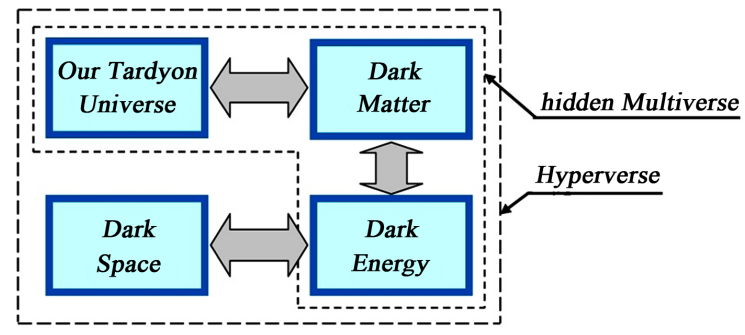

(a)

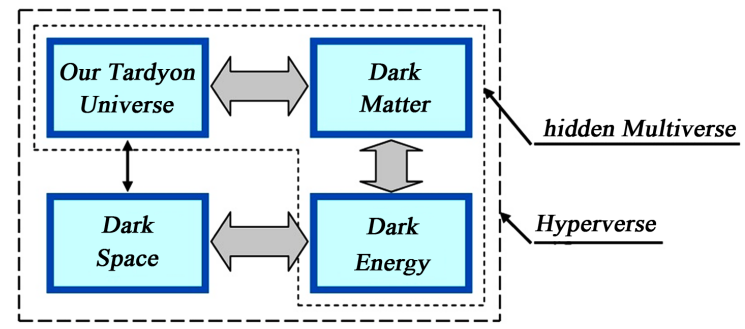

(b)

Figure 10. (Color online) Relative spatial location of our universe, as well as the universes of dark matter, dark energy and the dark space Multiverses. 
energy and dark space, there is an additional connection of our universe with one of the universes of the dark space. Therefore, information about dark matter can have some additional subtle, and therefore, perhaps not yet discovered, features that correspond to the properties of the dark space.

Thus, the hidden Multiverse can actually contain either twenty or twenty-one or twenty-two parallel universes. There are many structures, similar to those shown in Figures 5-9, i.e. consistent with the results of WMAP and Planck data analysis. However, experimental data available are not enough to clarify which of them is real. Therefore, additional astrophysical studies are needed.

\section{Verifiability of Hypothesis of the Hidden Multiverse}

Hypothesis of the hidden Multiverse considered in the article, in contrast to the large number of previously proposed unverifiable Multiverse hypotheses [76]-[84], is verifiable [85], because:

- It has experimental confirmation. That is dark matter and dark energy, which are actually other parallel universes of the hidden Multiverse, besides ours.

- Its another experimental confirmation is experiments at the Large Hadron Collider and other accelerators of subatomic particles, having shown that total mass of subatomic particles after acceleration is smaller than total mass of the particles before acceleration. The result can be explained by formation of tachyons due to acceleration and their transition to tachyon universes and antiverses through micro portals.

- The most convincing experimental evidence of the hidden Multiverse would be discovery of portals on the Earth and their geophysical research, as well as our subsequent penetration into adjacent parallel universes through them, if we are actually allowed by inhabitants of behind-the-portal supercivilizations [86].

Therefore, the proposed hypothesis of the hidden Multiverse has every reason to be called a theory.

\section{Conclusions}

Thus, the data obtained by WMAP and Planck spacecrafts turns out to not only determine the distribution of mass-energy between visible universe, dark matter and dark energy, but also to reveal the existence of dark space unknown to us so far.

Detection of indisputable evidence of dark space in the WMAP and Planck data has required an appropriate work on adequate interpretation of the data. Fallacy of certain fundamental provisions of the existing version of the STR, namely, the principle of light speed non-exceedance and the Monoverse hypothesis following there had to be proved. This, in turn, has required experimental studies in the theory of linear electric circuits, which, unlike the unsuccessful OPERA experiment, has definitely proved the validity of the general scientific principle of physical reality of imaginary numbers. Use of the principle of 
physical reality of imaginary numbers has proved fallacy of relativistic formulas of the existing version of the special theory of relativity.

Therefore, the adjusted relativistic formulas from which existence of the hidden Multiverse follows have been given in the article. The reason for mutual invisibility of parallel universes of the Multiverse has been explained. The phenomenon of dark matter and dark energy has been proved to be explained by existence of parallel universes. In particular, dark matter is explained by existence of other parallel universes adjacent to our universe, and dark energy is explained by existence of the rest parallel universes of the hidden Multiverse. In addition, the proposed hypothesis of the hidden Multiverse explains where antimatter is and why it does not annihilate with matter, as well as where tachyons are and why they do not violate the principle of causality. The hypothesis of the hidden Multiverse has been shown to be verifiable.

Mathematical analysis of WMAP and Planck spacecraft data has determined that the hidden Multiverse has a quaternion structure in six-dimensional space and contains twenty to twenty-two parallel universes; dark matter contains five or six parallel universes; and dark energy contains fourteen to sixteen parallel universes. Besides, the hidden Multiverse is connected through its portals to one-four other Multiverses, forming a new astrophysical entity, referred to as dark space. Dark space is much darker than dark matter and dark energy, since Multiverses contained therein are farther away from us than universes contained in dark energy. The hidden Multiverse and Multiverses of dark space form a Hyperverse.

\section{References}

[1] Ruiz-Lapuente, P., Ed. (2010) Dark Energy: Observational and Theoretical Approaches. Cambridge University Press, Cambridge.

[2] Amendola, L. and Tsujikawa, S. (2010) Dark Energy: Theory and Observations. Cambridge University Press, Cambridge.

[3] Bertone, G., Ed. (2013) Particle Dark Matter: Observations, Models and Searches. Cambridge University Press, Cambridge.

[4] Sanders, R.H. (2014) The Dark Matter Problem: A Historical Perspective. Cambridge University Press, Cambridge.

[5] Oort, J.H. (1932) Bulletin of the Astronomical Institutes of the Netherlands, 6, 249-287.

[6] Zwicky, F. (1933) Helvetica Physica Acta, 6, 110-127.

[7] Perlmutter, S. (2012) Reviews of Modern Physics, 84, 1127-1149.

[8] Schmidt, B.P. (2012) Reviews of Modern Physics, 84, 1151-1163.

[9] Riess, A.G. (2012) Reviews of Modern Physics, 84, 1165-1175.

[10] Weisstein, E.W. (2005) The CRC Concise Encyclopaedia of Mathematics. 3rd Edition, CRS Press, Roca Raton.

[11] Antonov, A.A. (2017) Norwegian Journal of Development of the International Science, 6, 50-63. http://www.njd-iscience.com

[12] Antonov, A.A. (2015) American Journal of Eelectrical and Electronics Engineering, 
3, 124-129.

[13] Antonov, A.A. (2015) Global Journal of Physics, 2, 145-149.

[14] Antonov, A.A. (2016) PONTE, 72, 131-142.

[15] Antonov, A.A. (2016) Journal of Modern Physics, 7, 2299-2313.

[16] Antonov, A.A. (2016) International Review of Physics, 10, 31-35.

[17] Antonov, A.A. and Bazhev, V.M. (1970) Means of Rising Deflecting Currents for Spiral Beam Sweep on the CRT Screen. USSR Patent No. 433650.

[18] Antonov, A.A. (2008) European Journal of Scientific Research, 21, 627-641.

[19] Antonov, A.A. (2009) European Journal of Scientific Research, 28, 193-204.

[20] Antonov, A.A. (2010) General Mathematics Notes, 1, 11-16.

[21] Antonov, A.A. (2010) International Journal of Pure and Applied Sciences and Technology, 1, 1-12.

[22] Antonov, A.A. (2010) American Journal of Scientific and Industrial Research, 1, 342-349.

[23] Antonov, A.A. (2013) International Journal of Management, IT and Engineering, 3, 219-230.

[24] Antonov, A.A. (2015) Journal of Russian Physical and Chemical Society, 87, 328-355.

[25] Antonov, A.A. (2015) General Mathematics Notes, 31, 34-53. http://www.emis.de/journals/GMN/yahoo_site_admin/assets/docs/4_GMN-9212-V 31N2.1293701.pdf

[26] Antonov, A.A. (2016) General Mathematics Notes, 35, 40-63. http://www.geman.in/yahoo_site_admin/assets/docs/4_GMN-10932-V35N2.318951 $\underline{46 . p d f}$

[27] Steinmetz, C.P. (2010) Theory and Calculation of Electric Circuit. Nabu Press, Charlstone.

[28] Frova, A. and Marenzana, M. (2006) Thus Spoke Galileo: The Great Scientist's Ideas and Their Relevance to the Present Day. Oxford University Press, NY.

[29] Einstein, A. (1920) Relativity: The Special and General Theory. H. Holt and Company, NY.

[30] Bohm, D. (2006) The Special Theory of Relativity. Routledge, Abingdon-onThames.

[31] Penrose, R. (2010) The Nature of Space and Time. Princeton University Press, Princeton.

[32] Larmor, J.J. (1897) Philosophical Transactions of the Royal Society A: Mathematical, Physical and Engineering Sciences, 190, 205-300.

[33] Lorentz, H.A. (1899) Proceeding of the Royal Netherlands Academy of Arts and Science, 1, 427-442.

[34] Poincaré, H. (1905) Comptes Rendus, 140, 1504-508.

[35] Einstein, A. (1905) Annalen der Physik, 17, 891-921.

[36] Adam, T., Agafonova, N., Aleksandrov, A., et al. (2011) Measurement of the Neutrino Velocity with the OPERA Detector in the CNGS Beam.

arXiv:1109.4897v4 [hep-ex]

[37] Antonello, M., Baibussinov, B., Boffelli, F., et al. (2012) Precision Measurement of the Neutrino Velocity with the ICARUS Detector in the CNGS Beam. 
arXiv:1208.2629 [hep-ex]

[38] Antonov, A.A. (2014) Global Journal of Science Frontier Research: A Physics \& Space Science, 14, 51-59.

[39] Antonov, A.A. (2014) American Journal of Scientific and Industrial Research, 5, 4052.

[40] Antonov, A.A. (2011) British Journal of Science, 2, 51-60.

[41] Antonov, A.A. (2012) Encyclopedia of Russian Thought. Reports to Russian Physical Society, 16, 3-20.

[42] Antonov, A.A. (2012) International Journal of Pure and Applied Sciences and Technology, 12, 43-56.

[43] Antonov, A.A. (2015) International Journal of Advanced Research in Physical Science, 2, 25-32.

[44] Antonov, A.A. (2012) American Journal of Scientific and Industrial Research, 3, 464-473.

[45] Antonov, A.A. (2016) Philosophy and Cosmology, 6, 11-27. http://ispcjournal.org/journals/2016-16/Antonov_16.pdf

[46] Chernobrov, V.A. (2000) Encyclopedia of Mysterious Places of the Earth. Veche, Moscow.

[47] Hinshaw, G., Larson, D., Komatsu, E., et al. (2013) Nine Year Wilkinson Anisotropy Probe (WMAP) Observations: Cosmological Parameter Results. arXiv: 1213.5226 [astro-ph/CO]

[48] Adam, R., Ade, P.A.R., Aghanim, N., et al. (2015) Plank 2015 Results. 1. Overview of Products and Scientific Results. arXiv:1502.01582v2 [astro-ph.CO]

[49] Antonov, A.A. (2015) International Journal of Physics, 3, 84-87.

[50] Antonov, A.A. (2015) Cosmology, 19, 40-61.

[51] Antonov, A.A. (2015) Global Journal of Science Frontier Research: A Physics and Space Science, 15, 33-38.

[52] Antonov, A.A. (2015) American Journal of Modern Physics, 4, 1-9.

[53] Antonov, A.A. (2015) Optics, 4, 43-47.

[54] Antonov, A.A. (2015) American Journal of Modern Physics, 4, 180-188.

[55] Antonov, A.A. (2016) Frontiers of Astronomy, Astrophysics and Cosmology, 2, 1-9.

[56] Antonov, A.A. (2016) Journal of Modern Physics, 7, 1228-1246.

[57] Antonov, A.A. (2016) Journal of Modern Physics, 7, 1933-1943.

[58] Antonov, A.A. (2017) Applied Physics Research, 9, 30-41.

[59] Antonov, A.A. (2017) Journal of Modern Physics, 8, 567-582.

[60] Feinberg, G. (1967) Physical Review, 159, 1089-1105.

[61] Bilaniuk, O.-M.P. and Sudarshan, E.C.G. (1969) Physics Today, 22, 43-51.

[62] Recami, E., Fontana, F. and Garavaglia, R. (2000) International Journal of Modern Physics, A15, 2793-2812.

[63] Tanaka, S. (1960) Progress of Theoretical Physics (Kyoto), 24, 171-200.

[64] Terletskyi, Y.P. (1966) Paradoxes in the Theory of Relativity. Nauka, Moscow.

[65] Hill, J.M. and Cox, B.J. (2012) Proceeding of Royal Society A: Mathematical, Physical and Engineering Sciences, 468, 4174-4192.

[66] Dirac, P.A.M. (1933) Theory of Electrons and Positrons. Nobel Lecture. 
[67] Alfvén, H. (1966) Worlds-Antiworlds: Antimatter in Cosmology. W. H. Freeman \& Co., San Francisco.

[68] Foot, R. (2002) Shadowlands: Quest for Mirror Matter in the Universe. Universal Publishers, Parkland.

[69] Frazer, G. (2004) Antimatter: The Ultimate Mirror. Cambridge University Press, Cambridge.

[70] Santilli, R.M. (2006) Isodual Theory of Antimatter: With Applications to Antigravity, Grand Unification and Cosmology. Springer Netherlands, Dordrecht.

[71] Alain, M. and Le Brun, V. (2012) Matter, Dark Matter, and Anti-Matter: In Search of the Hidden Universe. Springer-Verlag, NY.

[72] Antonov, A.A. (2016) PONTE, 72, 288-300.

[73] Kantor, I.L. and Solodovnikov, A.S. (1989) Hypercomplex Numbers. Springer-Verlag, Berlin.

[74] Antonov, A.A. (2015) Global Journal of Science Frontier Research: A Physics and Space Science, 15, 8-15.

[75] Antonov, A.A. (2017) Natural Science, 9, 43-62.

[76] Deutch, D. (1998) The Fabric of Reality: The Science of Parallel Universes and Its Implications. Penguin Books, NY.

[77] Greene, B. (2005) The Fabric of the Cosmos: Space, Time, and the Texture of Reality. Penguin, London.

[78] Kaku, M. (2006) Parallel Worlds: A Journey through Creation, Higher Dimensions, and the Future of the Cosmos. Anchor, NY.

[79] Vilenkin, A. (2006) Many Worlds in One: The Search for Other Universes. Hill and Wong, NY.

[80] Carr, B., Ed. (2009) Universe or Multiverse? Cambridge University Press, Cambridge.

[81] Gribbin, J. (2010) In Search of the Multiverse: Parallel Worlds, Hidden Dimensions, and the Ultimate Quest for the Frontiers of Reality. Wiley \& Sons, Inc., Hoboken.

[82] Greene, B. (2011) The Hidden Reality: Parallel Universes and the Deep Laws of the Cosmos. Vintage, NY.

[83] Stephen Hawking, S. and Mlodinow, L. (2012) The Grand Design. Reprint Edition, Bantam.

[84] Tegmark, M. (2014) Our Mathematical Universe: My Quest for the Ultimate Nature of Reality. Vintage, NY.

[85] Antonov, A.A. (2016) Global Journal of Science Frontier Research: A Physics and Space Science, 16, 4-12.

[86] Antonov, A.A. (2015) Cosmology. Commentaries: Stephen Hawking's Aliens. The Search for Intelligent Extraterrestrial Life. Project Breakthrough Listen. 